\title{
A pragmatic approach to measuring adherence in treatment delivery in psychotherapy
}

Marc Serfaty, Roz Shafran, Victoria Vickerstaff, Trefor Aspden.

Dr Marc Serfaty, Division of Psychiatry, University College London, London, UK. Prof Roz Shafran, UCL Great Ormond Street Institute of Child Health, London, UK. Dr Victoria Vickerstaff, Marie Curie Palliative Care Research Department, University College London, London, UK.

Dr Trefor Aspden, Division of Psychiatry, University College London, London, UK.

\section{Acknowledgements:}

We wish to thank the participants for supporting this study and therapists for taking part in the study and completing the TCC. We also wish to thank Dr S Singh for his independent ratings of therapy, Dr S Moorey and K Mannix for their preparation of the CanTalk treatment manual and help with developing the TCC and Dr A Gola for the Health Economics advice. We also wish to thank Professor D Veale for his helpful comments.

\section{Funding:}

This research was supported by a UK National Institute of Health Research grant (No. NIHR-HTA -09/33/02). 


\section{Abstract}

Background: Collecting measures of therapists' adherence to treatment manuals and therapists' competence is recommended for evaluating treatment integrity, yet the ways to do this are poorly defined, time consuming, costly and may be subject to rater bias.

Aims: To describe the development of a tool to measure adherence to manualised CBT, using a self-rated Therapy Component Checklist (TCC); to test its application in research and clinical practice; to determine its validity; and consider its cost benefits. Method: A randomised controlled trial in 230 people with advanced cancer evaluated the effectiveness of CBT for depression. In this, therapists were required to deliver a manualised treatment. Experts agreed on the key components of therapy and therapists were asked to record these at the end of each therapy session by ticking a TCC. Inter-rater reliability of the TCC was tested using an independent rater, blind to the therapists' responses.

Results: One hundred and twenty four therapists delivered 543 sessions of CBT. TCCs were completed in 293, of which 39 were assessed by the independent rater. Self-reported TCC data suggested a close adherence to the manual. Prevalenceadjusted and bias-adjusted kappa scores suggested substantial or perfect agreement, $(>0.60)$ in 38 out of $46(82.6 \%)$ items. Self-rating of adherence saved around £96 per rating.

Conclusions: A TCC for clinical and research purposes should be considered as a quick and cost effective way of evaluating the main components of therapy delivered. The TCC approach could be applied to other psychological treatments and potentially may help with linking therapeutic interventions with outcome.

Declaration of interest. Dr Serfaty is a member of the NIHR General Board. 


\section{Introduction}

One of the key objectives of psychological interventions is to help people gain a better understanding of the issues that are troubling them and help them work out new ways of approaching situations that they find difficult, as well as suggesting new methods to help them cope. In order to properly understand and evaluate the impact of such interventions, Lichstein (Lichstein, Riedel, \& Grieve, 1994) generated a taxonomy of three key processes - whether the treatment is delivered (delivery), understood by the client (receipt) and whether what the patient has received is acted on (enactment).

There are two important factors in treatment delivery which are of particular interest to researchers(Dinger, Zilcha-Mano, Dillon, \& Barber, 2014); firstly, therapist adherence and secondly, therapist competence.

1. Therapist adherence refers to the extent to which a therapist uses the specific techniques of a particular therapy approach. Adherence is important as it enables the delivery of therapy to be standardised through manualised treatments, so that interventions can be defined, replicated and measured.

2. Therapist competence is the degree of therapist skillfulness in treatment delivery. These factors then enable the association between competence and outcome to be examined.

Both therapist adherence and competence are essential to ensure treatment integrity, defined as the extent to which a treatment is delivered as intended (Fairburn and Cooper, 2011) which is especially important when conducting studies on the efficacy of a specific form of psychological intervention.

There is an assumption that high therapist competence is likely to be associated with advantageous outcomes with therapy. However, the relationship between therapist competence and symptom change is mixed and relatively weak (Webb, DeRubeis, \& Barber, 2010) (Branson, Shafran, \& Myles, 2015) (Strunk, DeRubeis, Chiu, \& Alvarez, 2007), and ways of measuring competence are highly resource intensive and relatively 
unreliable (Loades and Armstrong, 2016). Furthermore, whether particular elements predict outcome from treatment remains to be established.

We propose an alternative way of establishing adherence using a therapist reported measures rather than direct observation of sessions. The disadvantage of direct observation of sessions is that it is prohibitively time consuming, costly, reliant on the judgement of one or two 'expert' individuals and, by necessity, takes a relatively small sample of the sessions on which to base overall judgement. Therapists' self-rating is inherently more feasible to implement in clinical practice than rating of live or recorded sessions and may also be as valid as observer rating. By eliminating the need for someone to spend time observing sessions, self-rating is likely to deliver considerable cost-savings without losing any validity.

We have now conducted a number of trials in which we are developing and testing the use of a Therapy Components Checklist (TCC), which is a therapist-reported measure of adherence. The evaluation of the TCC described in this paper, was achieved from an embedded study within an NIHR funded Randomised Controlled Trial of a cognitive behavioural intervention for depression in advanced cancer (M. Serfaty et al., 2016). We present these data and the clinical and research applications.

\section{Aims:}

The aims of this paper are to:

1. Describe the development of a TCC, designed to measure adherence, operationalising the components of therapy delivered.

2. Evaluate the feasibility of using the TCC as a measure of adherence in a clinical and research setting.

3. Test the validity of the TCC as a measure of adherence.

4. Consider the economic cost-benefits of the TCC.

\section{Methods:}

Data were collected by therapists participating in a randomised controlled trial (RCT) of CBT for people with advanced cancer and depression; the CanTalk trial. The full protocol (M. Serfaty, et al., 2016) and study findings (Marc Serfaty et al., 2019) are available elsewhere. One hundred and twenty four high level (Level 3) IAPT 
therapists from 25 IAPT/Wellbeing services from across England participated in the study. Therapists were required to be at least 2 years post qualification in CBT and be accredited with the British Association of Behavioural and Cognitive Psychotherapists (BABCP). Therapists were given one day's training, with optional top up training, in how to apply their CBT skills to people with advanced cancer and taken through a training manual detailing the delivery of CBT. This training included time on how to complete the TCC with examples and the opportunity to ask questions.

\section{Context-specific CBT Treatment Manual:}

Therapists used a treatment manual (available from the lead author). This guide enabled therapists to apply and adapt their existing skills to be context-specific for people with advanced cancer and provided a step-by-step approach, used flexibly. Sessions consisted broadly of the following: Session 1: Assessing and introducing the cognitive-behavioural model. Session 2: Developing an understanding of problems within a cognitive framework. Session 3: Reviewing the formulation, identifying new insights/changes through guided discovery, identifying helpful vs 'unhelpful' thinking. Sessions 4-5: Reformulating success experiences, identifying triggers and developing new coping strategies through guided discovery. Session 6-7: Challenging thoughts and generating alternative 'helpful' ways of thinking. Session 8: Problem solving, checking that concepts are understood and realistic concerns addressed along with introducing 'worry time'. Session 9: Consolidating CBT strategies, prioritising problems and using worry management strategies. Session 10: Reviewing progress. Session 11: Conducting relapse prevention through reviewing difficulties, identifying achievements, promoting personal resilience. Session 12: Future planning by creating relapse prevention checklists, and planning for action if distress or unhelpful behaviours/thinking recurs. Details of the CanTalk intervention is described in Serfaty et al (M. Serfaty, et al., 2016) and the full manual is available from the lead author.

Therapy was delivered at an IAPT/Wellbeing centre or over the phone, providing 3 face to face sessions had occurred in the first instance. Where possible, sessions were digitally-recorded and the therapists were asked to complete the therapy components checklist (TCC), described below, at the end of each therapy session in order to minimise recall bias. The TCC detailed what elements of the intervention they 
believed they had delivered. The therapists were asked to upload these TCC along with a digital recording of the therapy session onto a secure server called Data Safe Haven, using encryption software. They were also asked to keep a copy of the TCC in their therapy notes.

We were aware that not all sessions could be digitally recorded; some therapy sessions took place over the telephone, some patients did not consent to their therapy sessions being recorded and some trusts would not allow any data files to be uploaded.

Delivery of therapy: adherence and competence:

Adherence to manualised treatment:

Development of the Therapy Components Checklist (TCC):

The use and the development of a TCC arose from previous work (Buszewicz et al., 2017; M. A. Serfaty et al., 2009) and was further developed by working with specialists in the field of CBT and cancer. We identified what we considered to be the core elements required in the delivery of the intervention. These core elements were operationalised in the treatment manual. Central to the components checklist, we wanted to ensure that the therapist adhered to both general CBT procedures (i.e. they used a combination of cognitive, behavioural and cognitive behavioural techniques) as well as specific issues related to our CBT intervention in cancer. The TCC in the CanTalk study covered 5 main areas (General Procedures, Behavioural, Cognitive, cognitive Behavioural and specific cancer topics). While this division into areas is somewhat arbitrary as cognitive change may lead to behaviour change and vice versa, it provides a useful means of categorising elements based on their predominant mechanisms of action. The TCC consisted of a total of 46 elements.

The checklist is shown in table 1

Table 1 HERE. 
Collection of the TCC:

One hundred and fifteen out of two hundred and thirty participants with a DSM-IV diagnosis of depressive disorder were randomised to up to 12 sessions of CBT. Once the trial was completed and the database closed, TCC data were analysed (see below).

Independent ratings of the TCC:

Digital recordings of therapy were independently rated by an accredited member of the British Association of Behavioural and Cognitive Psychotherapists, who was blind to the aims of this study. This rater had over 15 years of CBT practice in a range of settings and had been trained in using the Cognitive Therapy Scale (CTS) and Cognitive Therapy Scale-Revised (CTS-R;Blackburn et al., 2001). The CTS-R is a measure of the delivery of CBT (Shaw et al., 1999). This same independent rater was used to rate both the CTS-R and to independently rate the TCC.

We adopted a pragmatic approach and randomly selected at least 1 in 10 therapy sessions, stratified by the phase of therapy (early: session 1-4, mid: session 5-8, or late: sessions 9-12), to obtain a broad evaluation of treatment delivery. The independent rater also completed this checklist and the results were compared for agreement.

Analysis of TCC:

The analysis includes descriptive statistics of the various elements reported by therapists using the TCC. Calculations of the validity of the TCC were undertaken by comparing the therapists' self-report data for a particular session with the independent rater's assessment. For each component of therapy, we calculated agreement between the independent assessor and the therapist's own assessment of whether the component was covered, providing the four possible outcomes of: 1) both rate the component was delivered; 2) both rate the component was not delivered; 3) therapist but not observer rated the component as delivered; and 4) observer but not therapist rated the component as delivered. Using these possible agreement outcomes, we calculated the percentage agreement and the kappa coefficient. Due to the unbalanced distribution of the counts of the four possible outcomes, the prevalenceadjusted and bias-adjusted kappa (PABAK) (Chen, Faris, Hemmelgarn, Walker, \& 
Quan, 2009) was also applied for each component. Data were analysed using Excel 2013. A score ranging from $0.60-0.79$ suggests moderate and $0.80-1.00$ suggests strong/almost perfect agreement (McHugh, 2012).

Costs:

The TCC takes at most, 5 minutes to complete by the therapist. We have therefore costed for 5 minutes of time from a grade 7 IAPT therapist, including service add on costs. The cost is $£ 82.00$ (US\$106.60) per hour, therefore for 5 minutes of this is $£ 6.83$ (US\$ 8.88). The costs of independently rating adherence requires a rater to listen to the recording of a therapy session for at least 60 minutes, which at current market rates costs $£ 82$ (US\$106.60) per hour of digital recording; although it takes 60-120 minutes to rate a session for competence, we agreed a fixed fee of $£ 100.00$ (US\$130.00) per recording.

Therapist competence:

Therapist competence in Cognitive Therapy was measured using the 12-item (CTS-R Blackburn, et al., 2001)). Each item is rated from 0 to 6 on a visual analogue scale ranging from incompetent, through to novice, advanced beginner, competent, proficient and expert. The total score ranges between 0 and 72 . Therapists would be expected to achieve a minimum score of 36 , which is the standard criterion for competence within IAPT services. While the CTS-R has been shown to be sensitive to the change in competence in therapy delivery from attending an IAPT training program, evidence for a relationship between competence as assessed by the CTS$\mathrm{R}$ and patient outcomes is mixed and inconclusive (Branson et al., 2015).

\section{Ethics Approval:}

The investigators obtained ethical approval for the study from London -Camberwell St Giles NRES committee, Central London REC3 (ref 11/LO/0376) and obtained informed consent from all participants. The trial was registered with the ISRCTN (International Standard of Randomised Controlled Trials Number) - 07622709. 


\section{Results:}

\section{Treatment delivery:}

Out of a potential 1,380 therapy sessions (115 participants in CBT arm multiplied by 12 sessions), participants actually attended 543 sessions (39.9\%). Of these 543 sessions received, 174 sessions were audio-recorded. Mean number of sessions received was 4.7 (SD 4.9). TCCs were completed for 293 sessions, CTS-Rs were independently rated in 55 and independent ratings of both the TCC's and CTS-R's were available in 39.

Adherence to treatment manual:

Self-rated TCC from therapists were returned for 293 out of 543 (54\%) sessions delivered. The total number of the elements ticked by the therapist and the proportion these were reported, in percent, is shown on table 2. Guided discovery, activity scheduling, discussion about specific cancer topics, covering the impact of the physical illness and beliefs and expectations about the illness were the most common interventions. It also needs to be noted that general procedures were used in 9.9$19.5 \%$ of sessions, depending very much on the stage of therapy.

Reliability of therapists' self-rating using the TCC:

Of the 55 sessions rated independently by the observer, paired independent rater and therapists' self-report ratings were available for 39 TCCs. This was because therapists were not always able upload the TCC because of similar reasons previously reported in the methods. The TCC has 46 elements, therefore for 39 TCC's completed, 1,794 data points were available each with four possible options: 1) both therapist and observer can agree that a component was used; 2) both can agree a component was not used; 3 the therapist but not observer can state that a component was used; and 4) the observer but not therapist can state that the component was used. Frequencies of these for individual components are presented in Table 3, while the overall frequencies across components are provided in table 4. Data with Kappa scores of $\geq$ 0.61 is considered significant. 
Findings suggest that there was a strong relationship between therapists self-report and the independent rater's report for most of the components delivered or not delivered, with the majority of Kappa scores being $>0.60$ in 33/46 (71.7\%). Notably low Kappa agreement scores were for the items "Guided discovery" and "Impact of physical illness" (kappa 0.40 and 0.38 respectively, indicating little agreement). We would like to point out, that guided discovery, believed to be an essential component of CBT, was reported as delivered by therapists in $39.9 \%$ of sessions. However as shown, the observer's report suggested that therapists were indeed using more guided discovery than they reported. By contrast, therapists reported more discussion about the patients' physical illness than reported by the observer.

Therapist competence:

The independent evaluation of therapy rated 55 recordings of therapy which generated a mean CTS-R score of 47.6 (SD 13.8); upper end of the proficient range. Forty seven $(84 \%)$ out of 55 scored 36 or more on independent ratings of the CTS-R indicating that they were competent. The mean CTS-R score by phase of therapy was 47.9 (SD 10.6) for $n=21$ early sessions, 48.1 (SD18.8) for $n=19$ middle sessions and 46.7 (SD10.9) for $n=15$ late sessions.

Costs:

The CanTalk study was typical of research trials, in that at least 1 out of 10 therapy sessions were rated to assess therapist adherence (and competence). Using our paired data, in which 39 sessions were independently assessed the therapists' adherence using the TCC (and for competence using the CTS-R). The cost saving for self-rating $£ 6.83$ (US\$ 8.88) versus independent rating $£ 100$ (US\$130) is £93.17 (US\$ 121.21) per rating. The costs saving for the 39 sessions in a trial such as CanTalk would be $£ 3,633.63$ (US\$4,723.72).

\section{Discussion:}

To our knowledge, this is the first study to compare the reliability of a self-completed TCC with completion by an expert observer. Findings suggest that a self-rated measure of therapist adherence (TCC) could be operationalised and used both 
clinically and for research purposes. Adherence to the treatment manual was high with therapists reporting the use of a wide range of elements expected in the delivery of manualised CBT. Independent ratings of therapy, using the TCC, suggested that therapists' self-report, were an accurate reflection of adherence.

There were some discrepancies in self versus objective report of interventions undertaken. Overall discrepancies in inaccuracies in reporting were low, with $12 \%(212 / 1,794)$ of interventions reported by therapists not identified by the rater and $4.7 \%(85 / 1,794)$ of interventions identified by the rater not self-reported by the therapist.

Whilst the TCC enabled us to evaluate treatment delivery, it is not clear whether they were received by patients and then enacted on (Lichstein, et al., 1994). Evaluating these processes was beyond the scope of this work.

Therapist competence and adherence are distinct. This study suggests that self-report may be a valid way of assessing adherence. Although this study did not look at selfreported competence, self-report is less likely to be a valid measure of competence, with the literature currently suggesting that therapists tend to over- or under-report their own competence (Muse and McManus, 2013).

\section{Strengths}

The practical advantage of not engaging in complex, time-consuming, expensive and potentially unreliable procedures to evaluate adherence to therapy is clear. By selfmonitoring using the TCC, therapists may be changing their practice and adhering more closely to the treatment protocol which hopefully should be translated into better outcomes. Compared to an independent rater, the TCC enables more data to be collected and assessed across patients and stages of treatment, and at lower cost. Furthermore, as some patients choose not to have their therapy sessions recorded, as they may be concerned about confidentiality, the TCC provides a useful measure of treatment delivery. Until now there has been no way of establishing therapist adherence to protocol for such patients. Clinicians and their supervisors can use the TCC to review the components of therapy delivered and to consider whether elements missed in therapy should be included. 
Examining the relationship between the components used in therapy and outcome may clarify the elements and timing associated with therapeutic change. Importantly, independent ratings of therapy are expensive compared with the cost of self-report which is relatively small, and results in a saving of £93.17 (US\$121.21) per session. The problem with using random sampling methods, is that a crucial element of change may be missed. However, independently rating all therapy sessions would be so costly to be impractical. A benefit of the current approach is that asking therapists to self-rate adherence even for all 543 sessions delivered, would only cost $£ 3,708.69$ (US\$ 4,821.30) which is affordable, compared to prohibitive costs of independent ratings of $£ 54,300$ (US\$ 70570). As therapist self-report appears to be an accurate reflection of treatment delivery, the practical advantage and cost savings of therapist self-report is evident and applicable to a range of therapeutic approaches.

\section{Limitations:}

This study looked at checklists completed across multiple therapy sessions. Samples assessed were from a random selection of therapy sessions. In some cases, data were collected for sessions delivered by the same therapist, which introduces selection bias. This is a problem for most RCTs where there are more patients than therapists as this limits the psychometric evaluation of the TCC. The use of large data sets, such as IAPT, would enable researchers collect sufficient TCCs to ensure that large numbers TCCs were available to evaluate the psychometric properties of the TCC whilst at the same time ensuring that data are collected from different therapists, thus minimising bias. The reasons for therapists not completing the TCC and/or not recording and uploading recordings of therapy sessions may be associated with bias, for example, the therapist is concerned poor therapy was delivered. Though we did not formally record this, informal feedback indicated that therapists could not supply us with the information on line because of restrictions downloading software and limitations on data transfer from Trust computers to external sources. Nevertheless, the first step in measuring adherence remains to establish whether self-report is possible and whether consistent findings appear to be generated between observer and self-rater. Whilst resources were limited, it would be beneficial to assess inter-rater reliability of independent assessors of the TCC. 
As there were no significant improvement in depression scores with CBT in this population, it was not possible in this study to examine which components of therapy elicit change.

Now that we have demonstrated the feasibility of the TCC, the next step should include establishing the psychometric properties of the TCC in a large population, which would include dropping components which are correlated with each other and those which were infrequently used, thus generating an abbreviated checklist. We still do not know whether the components of therapy selected by experts will effect an improvement in the target symptoms.

The context of the setting in which the TCC is completed is important. For example, when delivering therapy over the telephone, non-verbal cues linking thoughts and feelings may be lost. This may in turn influence how therapy is delivered and what is recorded in the TCC. Caution is therefore required when applying the TCC to different therapeutic settings.

The reliance on one expert rater is a limitation and further work could include assessing interrater reliability when using the TCC. Athough self-ratings of competence are not recommended (Muse and McManus, 2013) a specific study aimed at comparing self ratings of competence with independent assessor ratings is not only consistent with our findings, but suggests if anything, self raters' tend to underestimate their skill (McManus, Rakovshik, Kennerley, Fennell, \& Westbrook, 2012).

\section{Implications:}

The study found that it was feasible for therapists to complete a TCC outlining the interventions used in a particular therapy session. Whilst the TCC was not used in supervision in the CanTalk study, adaptations have been applied to other studies, (e.g. CanACT (Low et al., 2016)), as a way of enabling supervisors to discuss potential deficits in treatment delivery. Collection of large TCC data sets would potentially help identify the components of therapy which are associated with a beneficial response to treatment. In turn this would facilitate briefer and more efficient interventions. The TCC may also enable comparison between therapists to see whether adherence to specific components is a better predictor of outcome than competence. Finally clients could also be asked to complete a shortened and simplified TCC which could be compared 
to the TCC to see whether Lichstein's (Lichstein, et al., 1994) second process, receipt of therapy, has occurred.

A self reported assessment of adherence to the protocol appears to be viable, cost effective and demonstrates at least good agreement with independent ratings of adherence. Further research is needed to more fully evaluate the reliability and validity of this therapistcompleted TCC. This TCC has potential to be used as a routine part of clinical practice during therapy delivery to prevent 'therapist drift' (Waller and Turner, 2016) and for clinical researchers interested in an efficient, cost-effective way of assessing adherence to their protocol. 


\section{Disclaimers:}

The report is independent research and the views expressed in this publication are those of the authors and not necessarily those of the NHS, the NIHR, or the Department of Health. 


\section{References}

Blackburn, I.-M., James, I. A., Milne, D. L., Baker, C., Standart, S., Garland, A., \& Reichelt, F. K. (2001). The revised cognitive therapy scale (CTS-R): psychometric properties. Behavioural and Cognitive Psychotherapy, 29(04), pp. 431-446.

Branson, A., Shafran, R., \& Myles, P. (2015). Investigating the relationship between competence and patient outcome with CBT. Behaviour Research and Therapy, 68, pp. 19-26. doi:10.1016/j.brat.2015.03.002

Buszewicz, M., Cape, J., Serfaty, M., Shafran, R., Kabir, T., Tyrer, P., . . Nazareth, I. (2017). Pilot of a randomised controlled trial of the selective serotonin reuptake inhibitor sertraline versus cognitive behavioural therapy for anxiety symptoms in people with generalised anxiety disorder who have failed to respond to lowintensity psychological treatments as defined by the National Institute for Health and Care Excellence guidelines. Health Technology Assessment (Winchester, England), 21(45), p 1.

Chen, G., Faris, P., Hemmelgarn, B., Walker, R. L., \& Quan, H. (2009). Measuring agreement of administrative data with chart data using prevalence unadjusted and adjusted kappa. BMC Medical Research Methodology, 9(1), p 5.

Dinger, U., Zilcha-Mano, S., Dillon, J., \& Barber, J. P. (2014). Therapist Adherence and Competence in Psychotherapy Research The Encyclopedia of Clinical Psychology: John Wiley \& Sons, Inc.

Fairburn, C. G., \& Cooper, Z. (2011). Therapist competence, therapy quality, and therapist training. Behaviour Research and Therapy, 49(6-7), pp. 373-378. doi:10.1016/j.brat.2011.03.005

Lichstein, K. L., Riedel, B. W., \& Grieve, R. (1994). Fair tests of clinical trials: A treatment implementation model. Advances in Behaviour Research and Therapy, 16(1), pp. 1-29.

Loades, M. E., \& Armstrong, P. (2016). The challenge of training supervisors to use direct assessments of clinical competence in CBT consistently: a systematic review and exploratory training study. The Cognitive Behaviour Therapist, 9

Low, J., Serfaty, M., Davis, S., Vickerstaff, V., Gola, A., Omar, R. Z., . . . Jones, L. (2016). Acceptance and commitment therapy for adults with advanced cancer (CanACT): study protocol for a feasibility randomised controlled trial. [journal article]. Trials, 17(1), p 77. doi:10.1186/s13063-016-1169-8 Retrieved from https://doi.org/10.1186/s13063-016-1169-8

McHugh, M. L. (2012). Interrater reliability: the kappa statistic. Biochemia medica, 22(3), pp. 276-282.

McManus, F., Rakovshik, S., Kennerley, H., Fennell, M., \& Westbrook, D. (2012). An investigation of the accuracy of therapists' self-assessment of cognitivebehaviour therapy skills. $\mathrm{Br} J$ Clin Psychol, 51(3), pp. 292-306. doi:10.1111/j.2044-8260.2011.02028.x

Muse, K., \& McManus, F. (2013). A systematic review of methods for assessing competence in cognitive-behavioural therapy. Clinical Psychology Review, 33(3), pp. 484-499. doi:https://doi.org/10.1016/j.cpr.2013.01.010 Retrieved from http://www.sciencedirect.com/science/article/pii/S0272735813000238

Serfaty, M., King, M., Nazareth, I., Moorey, S., Aspden, T., Tookman, A., . . Wood, J. (2019). Manualised cognitive behavioural therapy in treating depression in advanced cancer: the CanTalk RCT. Health Technology Assessment, 23(19), pp. 1-106. 
Serfaty, M., King, M., Nazareth, I., Tookman, A., Wood, J., Gola, A., . . . Jones, L. (2016). The clinical and cost effectiveness of cognitive behavioural therapy plus treatment as usual for the treatment of depression in advanced cancer (CanTalk): study protocol for a randomised controlled trial. Trials, 17(1), p 113. doi:10.1186/s13063-016-1223-6

Serfaty, M. A., Haworth, D., Blanchard, M., Buszewicz, M., Murad, S., \& King, M. (2009). Clinical effectiveness of individual cognitive behavioral therapy for depressed older people in primary care: a randomized controlled trial. Archives of General Psychiatry, 66(12), pp. 1332-1340.

Shaw, B. F., Elkin, I., Yamaguchi, J., Olmsted, M., Vallis, T. M., Dobson, K. S., .. . Imber, S. D. (1999). Therapist competence ratings in relation to clinical outcome in cognitive therapy of depression. Journal of Consulting and Clinical Psychology, 67(6), pp. 837-846. doi:10.1037/0022-006x.67.6.837

Strunk, D. R., DeRubeis, R. J., Chiu, A. W., \& Alvarez, J. (2007). Patients' competence in and performance of cognitive therapy skills: relation to the reduction of relapse risk following treatment for depression. Journal of Consulting and Clinical Psychology, 75(4), pp. 523-530. doi:10.1037/0022-006x.75.4.523

Waller, G., \& Turner, H. (2016). Therapist drift redux: Why well-meaning clinicians fail to deliver evidence-based therapy, and how to get back on track. Behaviour Research and Therapy, 77, pp. 129-137. doi:10.1016/j.brat.2015.12.005

Webb, C. A., DeRubeis, R. J., \& Barber, J. P. (2010). Therapist adherence/competence and treatment outcome: A meta-analytic review. Journal of Consulting and Clinical Psychology, 78(2), pp. 200-211. doi:10.1037/a0018912 


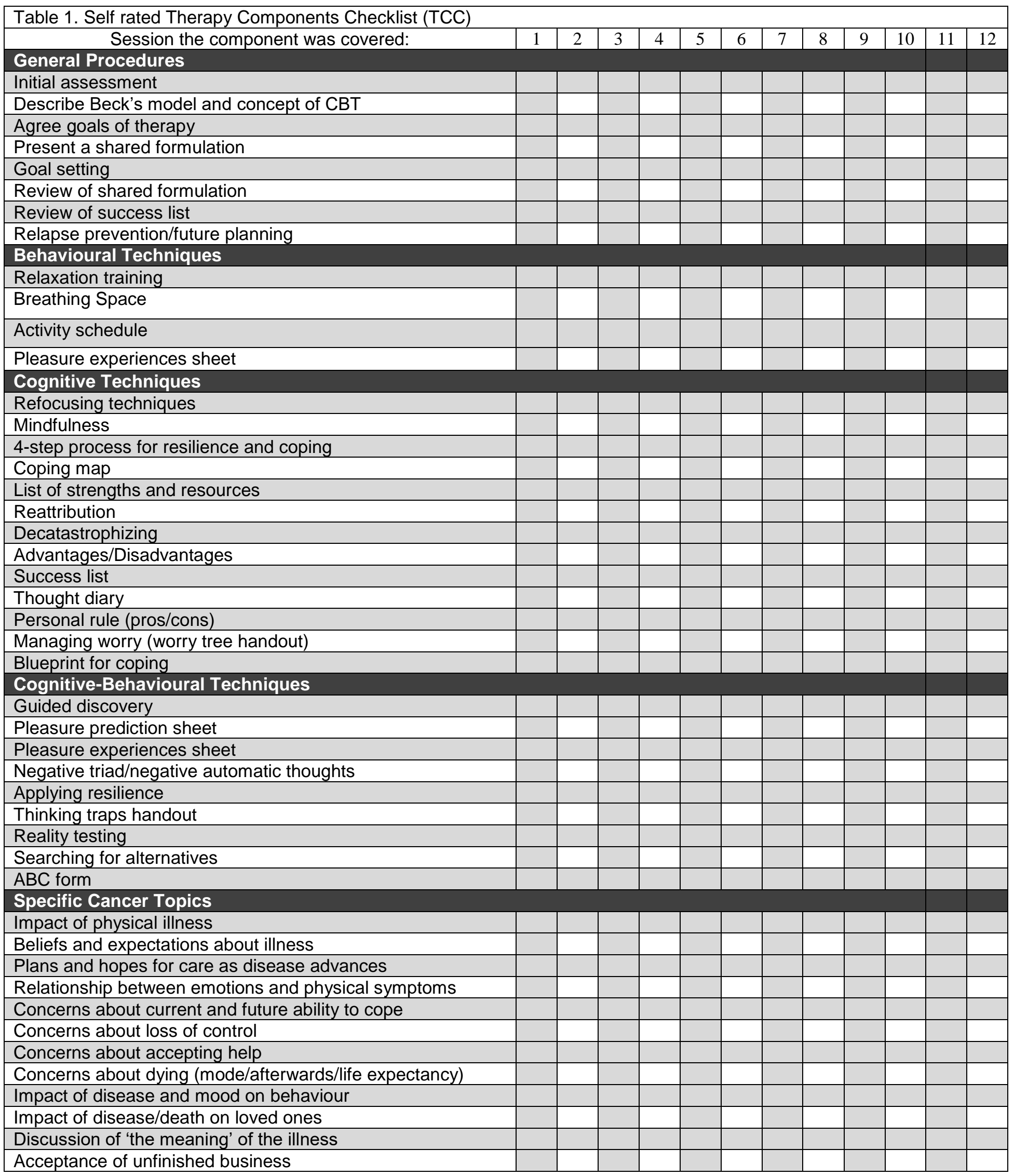


Table 2. Components of therapy delivered.

\begin{tabular}{|c|c|c|}
\hline Component & $\begin{array}{c}\text { Frequency } \\
\text { component was } \\
\text { used }^{\star}\end{array}$ & $\begin{array}{l}\text { Percentage of sessions } \\
\text { component used in }\end{array}$ \\
\hline General Procedures Total & 178 & $60.8 \%$ \\
\hline Initial assessment & 48 & $16.4 \%$ \\
\hline Describe Becks Model and concept of CBT & 47 & $16.0 \%$ \\
\hline Agree Goals of therapy & 57 & $19.5 \%$ \\
\hline Present a shared formulation & 35 & $11.9 \%$ \\
\hline Goal setting & 55 & $18.8 \%$ \\
\hline Review of shared formulation & 35 & $11.9 \%$ \\
\hline Review of success list & 29 & $9.9 \%$ \\
\hline Relapse prevention/Future planning & 33 & $11.3 \%$ \\
\hline Behavioural Techniques Total & 109 & $37.2 \%$ \\
\hline Relaxation Training & 21 & $7.2 \%$ \\
\hline Breathing space & 9 & $3.1 \%$ \\
\hline Activity Schedule & 86 & $29.4 \%$ \\
\hline Pleasure experiences sheet & 23 & $7.8 \%$ \\
\hline Cognitive Techniques Total & 168 & $57.3 \%$ \\
\hline Refocusing techniques & 25 & $8.5 \%$ \\
\hline Mindfulness & 12 & $4.1 \%$ \\
\hline 4-step process for resilience and coping & 18 & $6.1 \%$ \\
\hline Coping map & 12 & $4.1 \%$ \\
\hline List of strengths and resources & 47 & $16.0 \%$ \\
\hline Reattribution & 30 & $10.2 \%$ \\
\hline Decatastrophising & 30 & $10.2 \%$ \\
\hline Advantages/Disadvantages & 27 & $9.2 \%$ \\
\hline Success list & 26 & $8.9 \%$ \\
\hline Thoughts diary & 38 & $13.0 \%$ \\
\hline Personal rule (Pros/cons) & 26 & $8.9 \%$ \\
\hline Managing worry (worry tree handout) & 17 & $5.8 \%$ \\
\hline Blueprint for coping & 28 & $9.6 \%$ \\
\hline Cognitive-behavioural Techniques Total & 169 & $57.7 \%$ \\
\hline Guided discovery & 117 & $39.9 \%$ \\
\hline Pleasure prediction sheet & 8 & $2.7 \%$ \\
\hline Pleasure experiences sheet & 16 & $5.5 \%$ \\
\hline Negative triad/negative automatic thoughts & 46 & $15.7 \%$ \\
\hline Applying resilience & 43 & $14.7 \%$ \\
\hline Thinking traps hand-out & 36 & $12.3 \%$ \\
\hline Reality testing & 30 & $10.2 \%$ \\
\hline Searching for alternatives & 43 & $14.7 \%$ \\
\hline$A B C$ form & 16 & $5.5 \%$ \\
\hline
\end{tabular}




\begin{tabular}{|c|c|c|}
\hline Specific Cancer Topics Total & 205 & $70.0 \%$ \\
\hline Impact of physical illness & 123 & $42.0 \%$ \\
\hline Beliefs and expectations about illness & 104 & $35.5 \%$ \\
\hline Plans and hopes for care as disease advances & 58 & $19.8 \%$ \\
\hline Relationship between emotional and physical symptoms & 59 & $20.1 \%$ \\
\hline Concerns about current and future ability to cope & 75 & $25.6 \%$ \\
\hline Concerns about loss of control & 40 & $13.7 \%$ \\
\hline Concerns about accepting help & 57 & $19.5 \%$ \\
\hline Concerns about dying (mode/afterwards/life expectancy) & 40 & $13.7 \%$ \\
\hline Impact of disease and mood on behaviour & 85 & $29.0 \%$ \\
\hline Impact of disease/death on loved ones & 90 & $30.7 \%$ \\
\hline Discussion of 'the meaning' of the illness & 30 & $10.2 \%$ \\
\hline Acceptance of unfinished business & 8 & $2.7 \%$ \\
\hline
\end{tabular}

* Nb. Frequency that component was used out of 293 sessions for which therapist completed checklists were available 
Table 3. Therapist-rater agreement for components of therapy delivered.

\begin{tabular}{|c|c|c|c|c|c|}
\hline & $\begin{array}{l}\text { Ticked by } \\
\text { therapist } \\
\text { and } \\
\text { observer }\end{array}$ & $\begin{array}{l}\text { Not ticked by } \\
\text { therapist or } \\
\text { observer }\end{array}$ & $\begin{array}{l}\text { Ticked by } \\
\text { therapist, not } \\
\text { ticked by } \\
\text { observer }\end{array}$ & $\begin{array}{l}\text { Not ticked by } \\
\text { therapist, } \\
\text { ticked by } \\
\text { observer }\end{array}$ & $\begin{array}{l}\text { PABAK } \\
\text { score }\end{array}$ \\
\hline \multicolumn{6}{|l|}{ General Procedures } \\
\hline Initial assessment & 0 & 36 & 3 & 0 & 0.85 \\
\hline Describe Becks Model and concept of CBT & 5 & 31 & 3 & 0 & 0.85 \\
\hline Agree Goals of therapy & 3 & 29 & 5 & 2 & 0.64 \\
\hline Present a shared formulation & 1 & 29 & 4 & 5 & 0.54 \\
\hline Goal setting & 1 & 28 & 9 & 1 & 0.49 \\
\hline Review of shared formulation & 0 & 33 & 4 & 2 & 0.69 \\
\hline Review of success list & 1 & 32 & 6 & 0 & 0.69 \\
\hline Relapse prevention/Future planning & 5 & 32 & 1 & 1 & 0.90 \\
\hline \multicolumn{6}{|l|}{ Behavioural Techniques } \\
\hline Relaxation Training & 2 & 36 & 1 & 0 & 0.95 \\
\hline Breathing space & 1 & 37 & 1 & 0 & 0.95 \\
\hline Activity Schedule & 4 & 23 & 9 & 3 & 0.38 \\
\hline Pleasure experiences sheet & 0 & 37 & 2 & 0 & 0.90 \\
\hline \multicolumn{6}{|l|}{ Cognitive Techniques } \\
\hline Refocusing techniques & 1 & 35 & 2 & 1 & 0.85 \\
\hline Mindfulness & 0 & 37 & 2 & 0 & 0.90 \\
\hline 4-step process for resilience and coping & 0 & 32 & 6 & 1 & 0.64 \\
\hline Coping map & 0 & 34 & 2 & 3 & 0.74 \\
\hline List of strengths and resources & 0 & 28 & 7 & 4 & 0.44 \\
\hline Reattribution & 0 & 35 & 1 & 3 & 0.79 \\
\hline Decatastrophizing & 0 & 38 & 1 & 0 & 0.95 \\
\hline Advantages/Disadvantages & 1 & 35 & 3 & 0 & 0.85 \\
\hline Success list & 1 & 32 & 3 & 3 & 0.69 \\
\hline Thoughts diary & 1 & 29 & 6 & 3 & 0.54 \\
\hline Personal rule (Pros/cons) & 0 & 37 & 1 & 1 & 0.90 \\
\hline Managing worry (worry tree handout) & 1 & 34 & 2 & 2 & 0.79 \\
\hline Blueprint for coping & 1 & 31 & 4 & 3 & 0.64 \\
\hline \multicolumn{6}{|l|}{ Cognitive-behavioural techniques } \\
\hline Guided discovery & 9 & 11 & 5 & 14 & 0.03 \\
\hline Pleasure prediction sheet & 0 & 39 & 0 & 0 & 1.00 \\
\hline Pleasure experiences sheet & 0 & 37 & 1 & 1 & 0.90 \\
\hline Negative triad/negative automatic thoughts & 0 & 32 & 5 & 2 & 0.64 \\
\hline Applying resilience & 0 & 33 & 6 & 0 & 0.69 \\
\hline Thinking traps handout & 4 & 26 & 3 & 6 & 0.54 \\
\hline Reality testing & 3 & 29 & 2 & 5 & 0.64 \\
\hline Searching for alternatives & 0 & 33 & 4 & 2 & 0.69 \\
\hline$A B C$ form & 1 & 35 & 2 & 1 & 0.85 \\
\hline
\end{tabular}




\begin{tabular}{|c|c|c|c|c|c|c|}
\hline & $\begin{array}{l}\text { Ticked by } \\
\text { therapist } \\
\text { and } \\
\text { observer }\end{array}$ & $\begin{array}{l}\text { Not ticked by } \\
\text { therapist or } \\
\text { observer }\end{array}$ & $\begin{array}{l}\text { Ticked by } \\
\text { therapist, not } \\
\text { ticked by } \\
\text { observer }\end{array}$ & $\begin{array}{l}\text { Not ticked by } \\
\text { therapist, } \\
\text { ticked by } \\
\text { observer }\end{array}$ & $\begin{array}{l}\text { PABAK } \\
\text { score }\end{array}$ & $\begin{array}{l}\text { KAPPA } \\
\text { score }\end{array}$ \\
\hline Impact of physical illness & 2 & 18 & 15 & 4 & 0.03 & 0.03 \\
\hline Beliefs and expectations about illness & 5 & 21 & 10 & 3 & 0.33 & 0.33 \\
\hline Plans and hopes for care as disease advances & 2 & 27 & 10 & 0 & 0.49 & 0.49 \\
\hline Relationship between emotional/physical symptoms & 0 & 34 & 5 & 0 & 0.74 & 0.74 \\
\hline Concerns about current/future ability to cope & 0 & 27 & 12 & 0 & 0.38 & 0.38 \\
\hline Concerns about loss of control & 1 & 32 & 5 & 1 & 0.69 & 0.69 \\
\hline Concerns about accepting help & 1 & 31 & 6 & 1 & 0.64 & 0.64 \\
\hline Concerns about dying & 3 & 29 & 4 & 3 & 0.64 & 0.64 \\
\hline Impact of disease and mood on behaviour & 2 & 22 & 14 & 1 & 0.23 & 0.23 \\
\hline Impact of disease/death on loved ones & 3 & 24 & 10 & 2 & 0.38 & 0.38 \\
\hline Discussion of 'the meaning' of the illness & 1 & 33 & 4 & 1 & 0.74 & 0.74 \\
\hline Acceptance of unfinished business & 0 & 38 & 1 & 0 & 0.95 & 0.95 \\
\hline
\end{tabular}


Table 4: Frequencies of agreement or disagreement between therapists and independent raters

\begin{tabular}{|c|l|c|c|c|}
\hline \multicolumn{2}{|l|}{$\begin{array}{l}\text { Comparing Components } \\
\text { from Therapists' TCC and } \\
\text { Rater's of TCC. }\end{array}$} & \multicolumn{3}{|c|}{ Therapist } \\
\cline { 2 - 5 } & $\begin{array}{l}\text { Components } \\
\text { present }\end{array}$ & $\begin{array}{l}\text { Components } \\
\text { absent }\end{array}$ & TOTAL \\
\hline \multirow{3}{*}{ Rater } & $\begin{array}{l}\text { Components } \\
\text { present }\end{array}$ & 66 & 85 & 151 \\
\cline { 2 - 5 } & $\begin{array}{l}\text { Components } \\
\text { absent }\end{array}$ & 212 & 1,431 & 1,643 \\
\cline { 2 - 5 } & TOTAL & 278 & 1,516 & 1,794 \\
\hline
\end{tabular}

